\title{
Efficacité pollinisatrice de différents traitements sur 2 lignées de féverole de printemps (Vicia faba $L$ var equina Steudel), à des niveaux d'autofertilité différents, avec utilisation de diverses espèces de Bombus Latr (Hymenoptera: Apidae)
}

\author{
J Le Guen 1, J Mesquida 2, JS Pierre 2, G Morin 1, \\ JN Tasei 3 , 5 Carre ${ }^{3}$
}

\author{
1 Station d'amélioration des plantes; \\ 2 Laboratoire de zoologie, INRA, centre de recherches de Rennes, BP 29, 35650 Le Rheu; \\ 3 INRA, centre de recherches de Poitou-Charentes, laboratoire de zoologie, \\ 86600 Lusignan, France
}

(Reçu le 2 décembre 1992; accepté le 27 décembre 1992)

\begin{abstract}
Résumé - Dans une expérience sous cages, l'efficacité de la pollinisation de 3 espèces de bourdons (Bombus lapidarius, $B$ pascuorum et $B$ hortorum) a été comparée à celle de 3 traitements (déclenchement manuel, autopollinisation, considérée comme témoin, et pollinisation libre hors cages) sur 2 lignées de féverole (Vicia faba L) l'une autofertile (D-27) et l'autre peu autofertile (D-23). Les résultats ont montré que le rendement grainier de la lignée autofertile n'est pas affecté par les modalités de pollinisation. Au contraire, celui de la lignée non autofertile est très sensible à l'espèce de bourdon et nécessite l'intervention des insectes pour accroître significativement la nouaison et le rendement grainier. Le fait que la lignée autofertile soit indépendante des conditions de pollinisation montre que l'autofertilité chez la féverole pourrait être un moyen naturel de régulariser et de stabiliser le niveau des rendements mais non de les accroître.
\end{abstract}

Vicia faba / Bombus / pollinisation entomophile / autopollinisation / rendement grainier

\section{INTRODUCTION}

La féverole (Vicia faba L var equina Steudel) est une espèce autocompatible partiellement allogame, et de nombreux auteurs (Riedel et Wort, 1960; Wafa et
Ibrahim, 1960; Scriven et al, 1961; Watts et Marshall, 1961; Cooper, 1964; Free, 1966; Martyanova, 1967; Pritsch, 1971; Poulsen, 1971, 1973, 1975; Tasei, 1976; Link, 1990; Mesquida et al, 1990) ont montré qu'elle est tributaire de l'entomo- 
faune pollinisatrice pour sa production grainière.

Dans une étude antérieure (Mesquida et al, 1990), l'effet nul ou positif de la pollinisation par les insectes sur les nouaisons et les composantes du rendement de la féverole a été mis en évidence selon le degré d'autofertilité de la lignée. Lorsque l'autopollinisation est prépondérante, le déclenchement de la colonne staminale par un agent pollinisateur ne semble pas obligatoire et les visites d'insectes n'entraînent aucun effet positif. Dans le cas contraire, le déclenchement par l'intermédiaire d'un agent pollinisateur devient nécessaire et la présence d'insectes accroît notablement la nouaison et la productivité grainière.

Nous avons également montré, qu'en conditions de cages, les effets de la pollinisation sur les composantes du rendement et sur les taux d'allogamie de ces lignées n'étaient pas significativement différents lorsque les vecteurs de pollen étaient des bourdons (Bombus lapidarius $\mathrm{L}$ ) ou des abeilles domestiques (Apis mellifera $\mathrm{L}$ ) (Mesquida et al, 1990; Carre et al, 1991) bien qu'une tendance en faveur des bourdons ait alors été notée. Ceux-ci se distinguent d'ailleurs très nettement des abeilles domestiques, en particulier par leur plus grande aptitude à déclencher les fleurs de fabacées, leur plus grande vitesse de butinage (de l'ordre de 1,4 à 3,5 fois supérieurs, d'après Pritsch, 1971; Poulsen, 1973; Tasei, 1976), et aussi par leur plus long proboscis autorisant des visites des corolles profondes, telles que celles de la féverole.

Dans la présente étude, notre but a été de mesurer les différences de comportement en terme d'efficacité entre diverses espèces de bourdons au niveau de la for- mation progressive des organes reproducteurs et des composantes du rendement en grains. Un second objectif était de vérifier si l'autofertilité peut être considérée comme un moyen de stabiliser, voire d'accroître le niveau de rendement. Nous avons pour cela réalisé une expérimentation au champ sous cages comportant diverses modalités de pollinisation appliquées à 2 lignées.

\section{MATÉRIEL ET MÉTHODES}

Cette étude a été réalisée en 1991 sur le domaine INRA du centre de recherches de Rennes, Le Rheu en Ille-et-Vilaine.

Nous avons utilisé dans ces expériences une lignée de féverole de printemps autofertile (D27) et une lignée peu autofertile (D-23). Ces lignées, bien que non isogéniques sont cependant génétiquement très proches, et ont été obtenues par sélection à la station d'amélioration des plantes de Dijon.

Le schéma expérimental était un dispositif bloc équilibré à 6 traitements et 3 blocs. Les traitements étaient les suivants :

- cages avec $B$ lapidarius $L$;

- cages avec $B$ pascuorum Scopoli;

- cages avec $B$ hortorum L;

- cages avec déclenchement manuel;

- cages en autopollinisation spontanée $(=$ témoin);

- plantes en pollinisation libre hors cages.

Dans les cages avec déclenchement manuel*, toutes les fleurs formées ont systématiquement été déclenchées au même stade. Nous avions antérieurement vérifié qu'à ce stade, il y avait compatibilité entre le pollen et les stigmates (Le Guen, non publié). Les plantes en autopollinisation ont été laissées pendant toute la durée de leur floraison sous les cages, sans aucune intervention. Les plantes en pollinisation libre hors des cages constituaient le traitement témoin pour les taux d'avortement et la

\footnotetext{
* Le déclenchement manuel est l'action d'écarter les pétales de la fleur pour l'ouvrir et forcer le contact entre le pollen et le stigmate (en anglais : tripping).
} 
productivité en grains par plante. Ce traitement a été randomisé de la même façon que les autres traitements dans le dispositif expérimental, avec un même nombre de répétitions. Bien que nous n'ayons pas réalisé la mesure de la faune pollinisatrice "naturelle", nous avons conservé ce traitement dans l'analyse statistique car il constitue, pour le généticien, la référence aux conditions naturelles.

Les cages avec bourdons contenaient chacune 2 fondatrices introduites dès le début de la floraison, ainsi que 2 caisses de nidification en bois de $25 \times 25 \times 25 \mathrm{~cm}$, selon le modèle et les matériaux décrits par Pouvreau (1965), Pouvreau et Marilleau (1977). Nous avons utilisé des cages grillagées avec une maille de $2 \mathrm{~mm}$. Les cages avaient une dimension de $3 \times 3 \times 2 \mathrm{~m}$ et chacune d'elles comportait 2 lignes de 10 plantes de la lignée autofertile D-27 adjacentes à 2 lignes de 10 plantes chacune de la lignée peu autofertile D-23. Les espaces entre les lignes étaient de $0,5 \mathrm{~m}$ et les distances entre plantes sur la ligne de $0,3 \mathrm{~m}$. À l'intérieur des cages avec bourdons, on a maintenu en permanence un complément d'aliments dans des nourrisseurs (miel, miel + eau à $50 \%$ et pollen). Notons que nous avons utilisé des fondatrices en période de nidification, dont on sait qu'une seule d'entre elles, ou éventuellement 2 , suffit à produire une activité pollinisatrice satisfaisante dans les cages décrites ici, utilisées aussi bien pour la pollinisation de la féverole (Berthelem, 1966) que pour celle du colza (Lerin, 1982; Mesquida et al, 1988). L'efficacité de l'activité des bourdons a été vérifiée sur la quantité d'organes reproducteurs produits (fleurs, gousses), les taux d'avortement des fleurs et des gousses, les taux de nouaison et les composantes du rendement. La production des organes reproducteurs a été notée à chaque nœud au fur et à mesure de leur apparition et de leur développement. Par souci de simplification, il a été convenu de classer les organes reproducteurs en 3 stades :

- stade 0 pour les fleurs;

- stade I pour les gousses de longueur inférieure ou égale à $2 \mathrm{~cm}$, dans lesquelles il n'est pas encore possible de déceler au toucher la présence de grains;

- stade II pour les gousses de plus de $2 \mathrm{~cm}$ de long, dans lesquelles les grains sont formés.

Les dénombrements de fleurs et de gousses des stades I et II ont été réalisés tous les 2 j sur
90 plantes par lignée (5 plantes par traitement et par répétition), préalablement prises au hasard et marquées. Les taux de nouaison et les taux d'avortement des fleurs et des gousses ont été calculés selon Mesquida et al (1990). Les composantes du rendement retenues sont le nombre de grains produits par plante et le poids de 1000 grains. Par ailleurs le rendement en grains par plante a été analysé.

Pour ce qui concerne l'analyse des données, les comparaisons de moyennes ont été réalisées sur les valeurs moyennes des 5 plantes par répétition. Nous avons utilisé le logiciel STAT.ITCF (Gouet et al, 1985), selon un modèle factoriel à 2 facteurs à effets fixés (traitements et génotypes). Dans le cas de rejet de l'hypothèse nulle, l'analyse de la variance a été suivie du test de classification des moyennes de Newman et Keuls au seuil de risque de $5 \%$. Les proportions ont été analysées après transformation arc sin $\sqrt{ }$. L. L'analyse de la variance selon un modèle factoriel complet n'a pas permis de mettre en évidence d'effet significatif pour l'interaction variété/traitement, quelle que soit la variable analysée. Dans la suite de cette note, nous ne considèrerons donc que les variances "génotypes" (ou lignée) et les variances "traitement".

\section{RÉSULTATS}

\section{Influence du traitement de pollinisation sur la production de fleurs et de gousses par plante entière}

Les traitements n'ont eu aucune action sur les quantités de fleurs totales produites par les plantes (tableau I), mais le fleurs produites par la lignée autofertile D-27 sont plus nombreuses que celles produites par la lignée peu autofertile (127 et 84 fleurs par plante respectivement, $P<0,05)$. La lignée autofertile a produit en moyenne et en conditions de cages avec bourdons, significativement plus de gousses au stade I que la lignée peu autofertile (14 à $22 \%$ en plus), mais moins (17 à 20\%) de gousses 
Tableau I. Nombre moyen de fleurs, de gousses au stade I et de gousses au stade II par plante en fonction des traitements chez les lignées D-27 et D-23.

\begin{tabular}{|c|c|c|c|c|c|c|c|c|}
\hline & & $\begin{array}{c}\text { B } \\
\text { lapidarius }\end{array}$ & $\begin{array}{c}\mathrm{B} \\
\text { pascuorum }\end{array}$ & $\underset{\text { hortorum }}{\text { B }}$ & $\begin{array}{l}\text { Déclenchement } \\
\mathrm{n} \text { manuel }\end{array}$ & $\begin{array}{l}t \text { Auto- } \\
\text { pollinisation }\end{array}$ & Extérieur & Moyenne \\
\hline $\begin{array}{l}\text { Nombre } \\
\text { de } \\
\text { fleurs }\end{array}$ & $\begin{array}{l}\mathrm{D}-27 \\
\mathrm{D}-23 \\
\text { Moyenne }\end{array}$ & $\begin{array}{c}129,5 \\
85,1 \\
107,3^{\mathrm{a}}\end{array}$ & $\begin{array}{c}124,3 \\
82,9 \\
103,6^{a}\end{array}$ & $\begin{array}{c}128,9 \\
84,8 \\
106,8^{a}\end{array}$ & $\begin{array}{c}123,5 \\
84,4 \\
103,9 a\end{array}$ & $\begin{array}{c}132,2 \\
83,4 \\
107,8^{a}\end{array}$ & $\begin{array}{c}126,5 \\
83,7 \\
105,1^{\mathrm{a}}\end{array}$ & $\begin{array}{r}127,5^{\mathrm{a}} \\
84,1^{\mathrm{b}}\end{array}$ \\
\hline $\begin{array}{l}\text { Nombre } \\
\text { de gousses } \\
\text { Stade I }\end{array}$ & $\begin{array}{l}\text { D-27 } \\
\text { D-23 } \\
\text { Moyenne }\end{array}$ & $\begin{array}{l}76,2^{a} \\
66,8 a \\
71,5 a\end{array}$ & $\begin{array}{l}72,7^{a} \\
59,5^{a} \\
66,1^{a}\end{array}$ & $\begin{array}{l}75,9^{a} \\
63,3^{a} \\
69,6^{a}\end{array}$ & $\begin{array}{l}78,7^{\mathrm{a}} \\
54,3^{\mathrm{a}} \\
66,5^{\mathrm{a}}\end{array}$ & $\begin{array}{l}68,4^{\mathrm{a}} \\
34,8^{\mathrm{b}} \\
51,6^{\mathrm{b}}\end{array}$ & $\begin{array}{l}58,1^{a} \\
58,0^{a} \\
58,1^{\text {ab }}\end{array}$ & $\begin{array}{l}71,7^{a} \\
56,1\end{array}$ \\
\hline $\begin{array}{l}\text { Nombre } \\
\text { de gousses } \\
\text { Stade II }\end{array}$ & $\begin{array}{l}\text { D-27 } \\
\text { D-23 } \\
\text { Moyenne }\end{array}$ & $\begin{array}{l}38,7^{a b} \\
45,3^{a} \\
42,0^{a}\end{array}$ & $\begin{array}{l}37,7^{a b} \\
45,4^{a} \\
41,5^{a}\end{array}$ & $\begin{array}{l}39,9^{a b} \\
48,0^{a} \\
43,9^{a}\end{array}$ & $\begin{array}{l}40,3^{a b} \\
35,7^{a b} \\
38,0^{a}\end{array}$ & $\begin{array}{l}36,8^{\mathrm{ab}} \\
11,1^{\mathrm{d}} \\
24,0^{\mathrm{b}}\end{array}$ & $\begin{array}{l}24,5^{c} \\
27,3^{b c} \\
25,9^{b}\end{array}$ & $\begin{array}{l}36,3^{a b} \\
35,5^{a b}\end{array}$ \\
\hline
\end{tabular}

Au sein d'une ligne, les moyennes affectées d'une même lettre ne sont pas significativement différentes au seuil de probabilité de $5 \%$.

au stade II. Le tableau I montre aussi qu'en autopollinisation les quantités de gousses produites par la lignée autofertile (stades I et II) sont équivalentes à celles obtenues dans les autres traitements sous cages. Au contraire, la lignée peu autofertile présente une baisse de production de fruits de plus de $70 \%$.

\section{Influence du traitement de pollinisation sur la production et la répartition des fleurs et des gousses par étage}

\section{Les fleurs}

L'analyse statistique montre que la répartition des fleurs par étage ne dépend pas des conditions de traitement. Les courbes de distribution sont, pour chaque lignée, identiques, unimodales et suivent le même rythme de production de fleurs par niveau florifère (fig 1). Les productions optimales de fleurs se situent au niveau des 10 premiers étages, avec un maximum situé au $5^{e}$ nœud florifère.

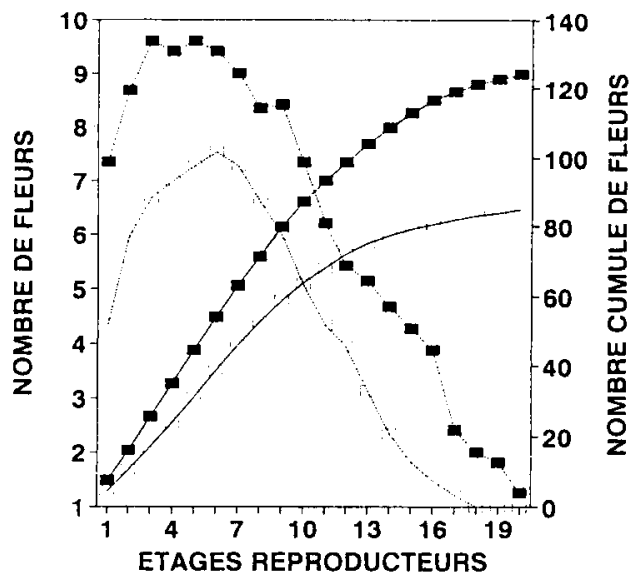

Fig 1. Évolution du nombre de fleurs en fonction du niveau de l'étage reproducteur (déclenchement manuel). $\rightarrow$ - D-27 cumul; ........ D-27

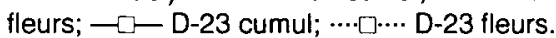

\section{Les gousses aux stades I et II}

La lignée autofertile D-27 apparaît peu sensible aux diverses conditions de traite- 
a

COURBES CUMULEES DES GOUSSES STADE 1 LIGNEE AUTOFERTILE (D-27)

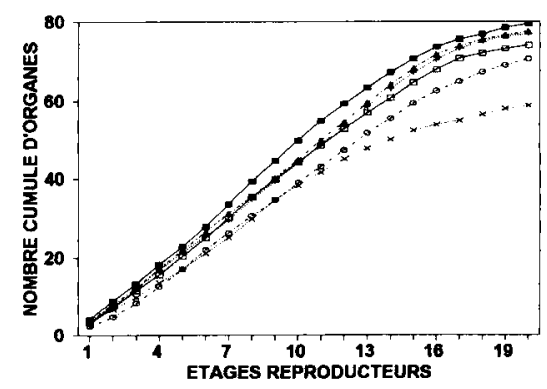

b

COURBES CUMULEES DES GOUSSES STADE ॥ LIGNEE AUTOFERTILE (D-27)

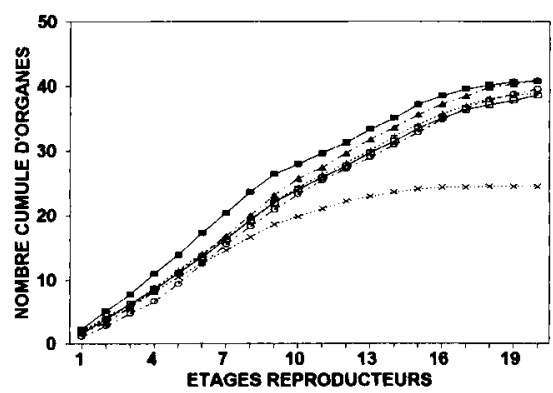

C COURBES CUMULEES DES GOUSSES \&TADE I LIGNEE PEU AUTOFERTILE (D-23)

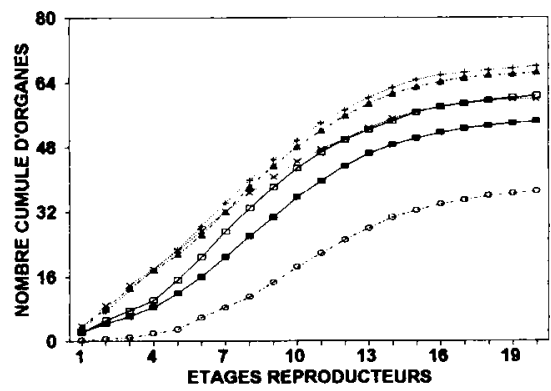

d COURBES CUMULEES DES GOUSSES STADE II LIGNEE PEU AUTOFERTILE (D-23)

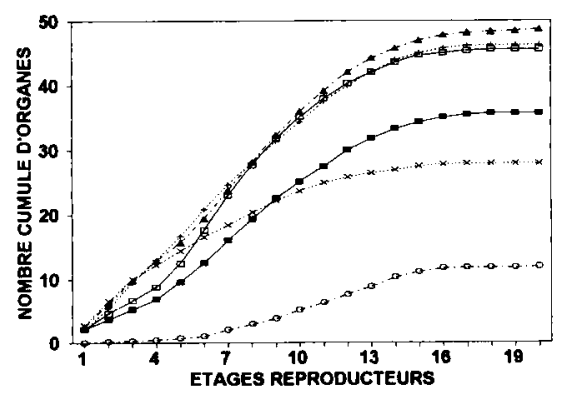

Fig 2. Courbes cumulées des gousses aux stades I et II en fonction du niveau de l'étage reproducteur. - $\square-$ Bombus lapidarius L; - - Bombus pascuorum Scopoli; - - - Bombus hortorum L; - - déclenchement manuel; - -0 - autopollinisation: ---X--- pollinisation extérieure.

ment ainsi qu'aux différentes espèces de bourdons. Les courbes cumulées correspondant aux différents traitements se supperposent (si l'on excepte le traitement pollinisation extérieure), aussi bien pour les gousses du stade I que du stade II (fig $2 \mathrm{a}$ et $2 b)$.

En revanche, la lignée peu autofertile D23 apparaît sensible aux conditions de pollinisation (fig $2 c$ et $2 d$ ), surtout pour ce qui concerne les gousses au stade II (fig 2d). Dans ce dernier cas, 3 types de réponses peuvent être notées:
- le traitement autopollinisation conduit à une courbe cumulée très faible, avec un maximum de 10 gousses au stade II produites pour l'ensemble des nœuds reproducteurs;

- les traitements déclenchement manuel et pollinisation extérieure présentent des courbes cumulées intermédiaires, avec un maximum de 30 à 35 gousses au stade II produites;

- enfin, les 3 traitements bourdon, statistiquement identiques entre eux avec une production maximale, quelle que soit l'es- 
pèce de bourdon, de 50 gousses par plante au stade II.

Si l'on considère l'évolution de la distribution des gousses (stades I et II) le long de la tige, on constate que celle-ci est à la fois fonction des génotypes et fonction des traitements (figs 3 et 4). La lignée autofertile D-27 (fig 3) présente des courbes globalement semblables, avec un plateau plus ou moins régulier entre les étages 2 et 15 , quel que soit le type de pollinisation.

Chez la lignée peu autofertile D-23 (fig 4), la distribution des organes reproducteurs est différente de la précédente, en ce sens qu'elle présente généralement une courbe "en cloche" avec des productions maximales dans la partie moyenne des tiges. Par ailleurs, la figure 4 montre que les différentes espèces de Bombus ont des comportements différents.

$B$ pascuorum (fig 4a) présente une efficacité faible sur la base des tiges (étages reproducteurs 1 à 4) et un accroissement rapide au-delà. Cette activité est comparable, quant à sa cinétique, à celle que l'on observe avec le traitement déclenchement manuel (fig 4d), bien que dans ce dernier traitement la production n'atteigne pas celle obtenue avec $B$ pascuorum.

$B$ hortorum et $B$ lapidarius (fig $4 b$ et $4 c$ ) présentent au contraire une activité plus grande sur les premiers étages, suivie d'une baisse sensible d'efficacité sur les étages 5 à 7 . Leur activité devient par la suite comparable à celle notée chez $B$ pascuorum. Les profils de courbes de $B$ hortorum et de $B$ lapidarius sont à rapprocher de ce que l'on observe en conditions de pollinisation extérieure (fig 4e), du moins pour les premiers étages reproducteurs.

L'autopollinisation (fig 4f) enfin se distingue des traitements précédents par une production moyenne nettement plus faible et une nouaison pratiquement nulle sur les 4 premiers nœuds reproducteurs.

\section{Entre lignées et dans les mêmes conditions de traitement}

Les différences entre lignées sont importantes.

En autopollinisation, la lignée peu autofertile D-23 (fig 4f) a produit en moyenne nettement moins de gousses que la lignée autofertile (fig $3 f$ ) et les courbes présentent une allure différente. Celles de la lignée peu autofertile font apparaître une faible production à la base de la tige (moins d'une gousse par étage en moyenne dans les étages 1-5). Cette production augmente brusquement ensuite dans les parties moyennes (étages 6-12), avec un maximum situé au $10^{\mathrm{e}}$ étage.

Chez la lignée autofertile (fig 3f), la production de gousses est d'emblée plus importante que celle de D-23 à la base de la tige (étages 1-5) et cette production se maintient relativement constante jusqu'à l'étage 15 , avec un maximum au $6^{\mathrm{e}}$ étage. Dans les cages avec $B$ hortorum et celles avec $B$ lapidarius, la lignée autofertile (fig $3 b$ et $3 c$ ) a produit nettement moins de gousses que D-23, essentiellement dans la partie moyenne des tiges (étages 7 à 13). Dans les cages avec $B$ pascuorum, la production de gousses de D-27 (fig 3a) est supérieure à celle de la lignée peu autofertile dans les étages 1-5 et 13-20.

\section{Influence du traitement de pollinisation sur les taux d'avortement et les taux de nouaison}

Le tableau II indique que le comportement des 2 lignées étudiées est différent. L'analyse de la variance fait apparaître un effet génotype et un effet traitement significatifs.

Pour ce qui concerne l'effet génotype, la lignée autofertile D-27 présente des taux d'avortement significativement supérieurs à ceux de la lignée peu autofertile D-23. II 


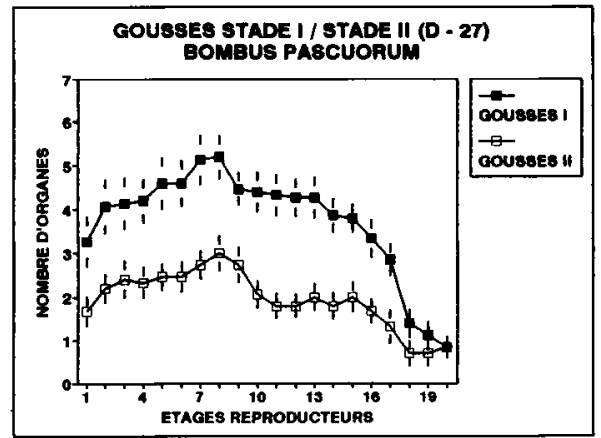

(a)

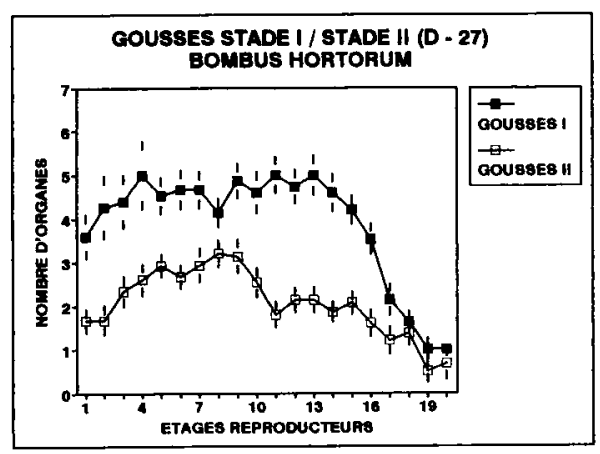

(b)

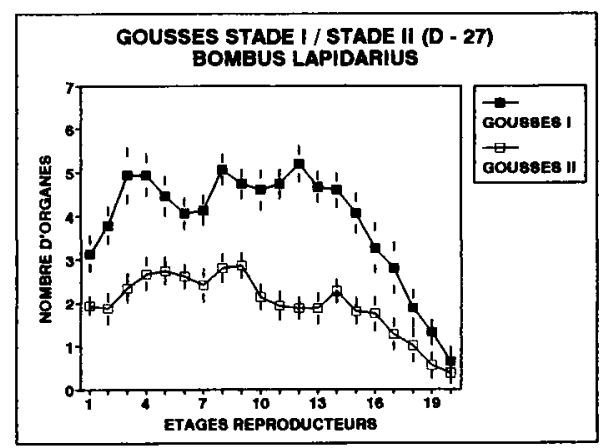

(c)

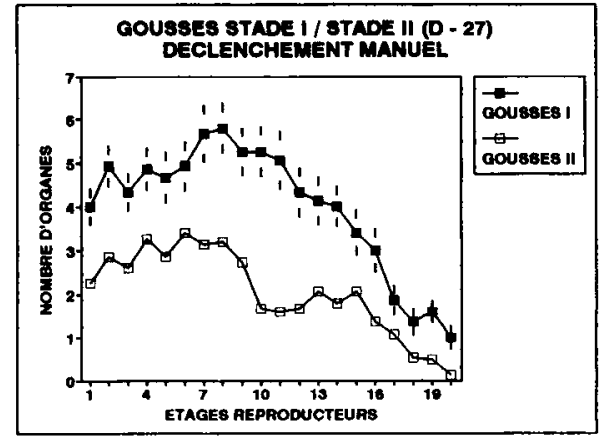

(d)

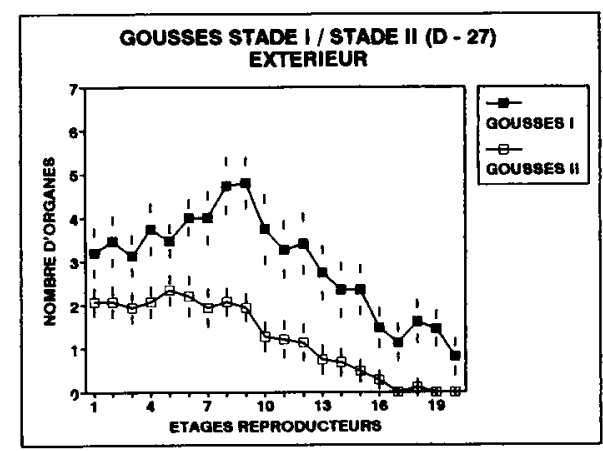

(e)

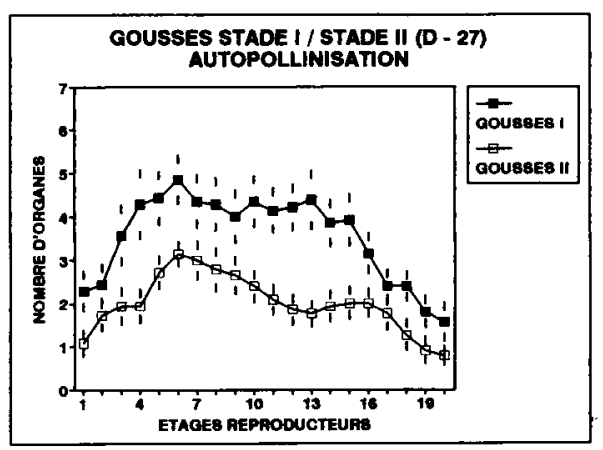

(f)

Fig 3. Évolution du nombre de gousses aux stades I et II en fonction de l'étage reproducteur pour les différents traitements chez la lignée autofertile D-27. 


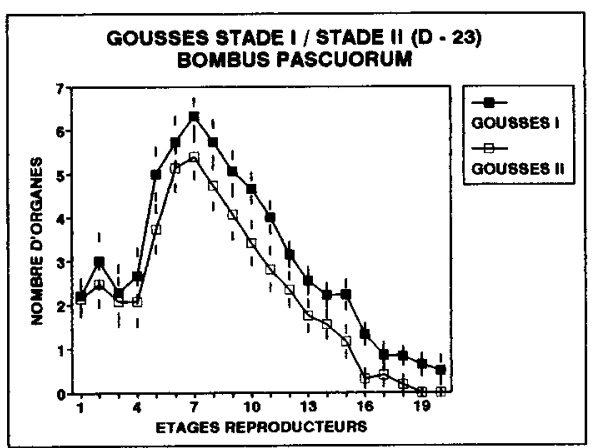

(a)

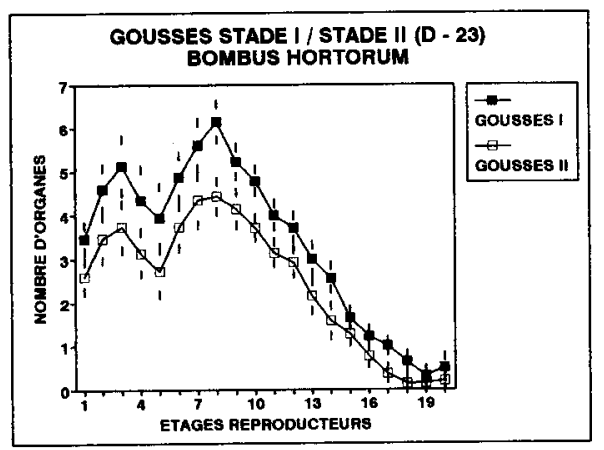

(b)

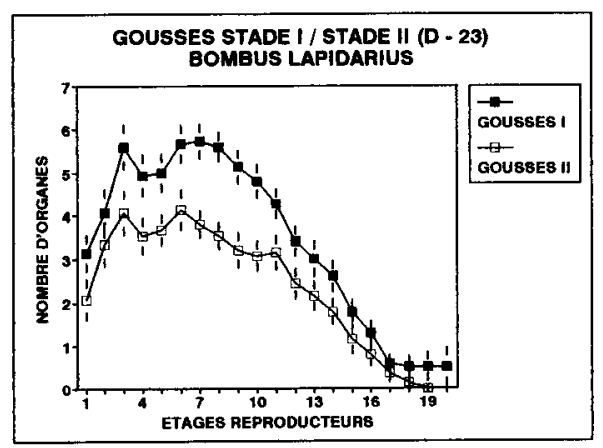

(c)

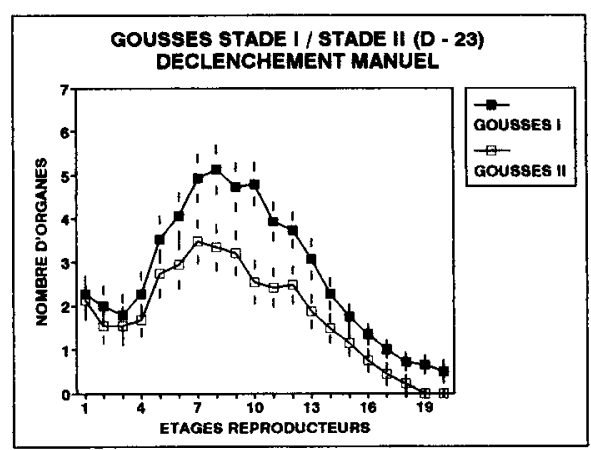

(d)

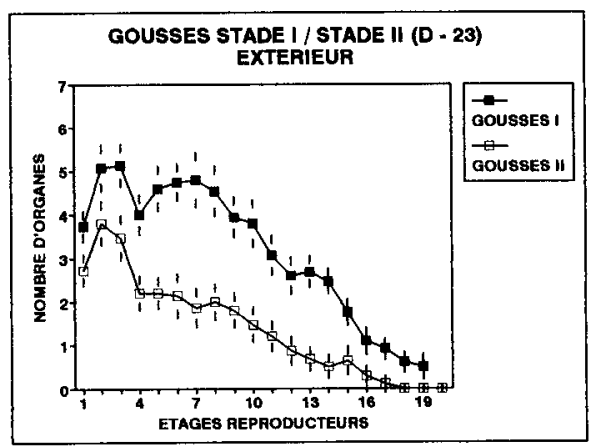

(e)

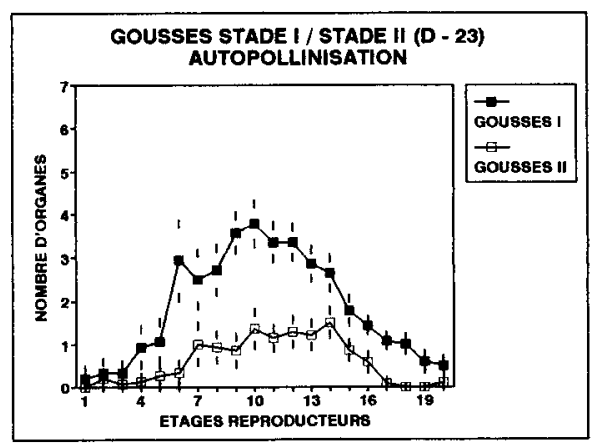

(f)

Fig 4. Évolution du nombre de gousses aux stades I et II en fonction du niveau de l'étage reproducteur pour les différents traitements chez la lignée peu autofertile D-23. (Pour l'ensemble des figures, sauf la figure 2, les points sur les courbes sont représentés par leur moyenne encadrée de l'écart type). 
Tableau II. Taux moyens d'avortement des fleurs et des gousses au stade l et taux moyens de nouaison des lignées $\mathrm{D}-27$ et $\mathrm{D}-23$ en fonction des traitements.

\begin{tabular}{|c|c|c|c|c|c|c|c|c|}
\hline & & $\begin{array}{c}\text { B } \\
\text { lapidarius }\end{array}$ & $\begin{array}{c}\text { B } \\
\text { pascuorum }\end{array}$ & $\underset{\text { hortorum }}{\mathrm{B}}$ & $\begin{array}{l}\text { Déclenchement } \\
\mathrm{n} \text { manuel }\end{array}$ & $\begin{array}{l}\text { Auto- } \\
\text { pollinisation }\end{array}$ & Extérieur & Moyenne \\
\hline $\begin{array}{l}\text { Taux } \\
\text { avortement } \\
\text { Fleurs }\end{array}$ & $\begin{array}{l}\text { D-27 } \\
\text { D-23 } \\
\text { Moyenne }\end{array}$ & $\begin{array}{l}41,1^{\mathrm{abc}} \\
21,5^{c} \\
31,3^{\mathrm{b}}\end{array}$ & $\begin{array}{l}42,2^{a b c} \\
28,0^{b c} \\
35,1^{b}\end{array}$ & $\begin{array}{l}40,7^{a b c} \\
14,4^{d} \\
27,5^{b}\end{array}$ & $\begin{array}{l}36,3^{a b c} \\
35,8^{a b c} \\
36,1^{b}\end{array}$ & $\begin{array}{l}46,8^{a b c} \\
57,6^{a} \\
52,2^{a}\end{array}$ & $\begin{array}{l}54,2^{a} \\
31,4^{b c} \\
42,8^{a b}\end{array}$ & $\begin{array}{l}43,6^{a} \\
31,5^{b}\end{array}$ \\
\hline $\begin{array}{l}\text { Taux } \\
\text { avortement } \\
\text { gousses } \\
\text { Stade I }\end{array}$ & $\begin{array}{l}\text { D-27 } \\
\text { D-23 } \\
\text { Moyenne }\end{array}$ & $\begin{array}{l}49,0^{\mathrm{bc}} \\
32,2^{\mathrm{d}} \\
40,6^{c}\end{array}$ & $\begin{array}{l}48,3^{b c} \\
23,7^{e} \\
36,0^{c}\end{array}$ & $\begin{array}{l}47,0^{c} \\
24,6^{e} \\
35,8^{c}\end{array}$ & $\begin{array}{l}48,7^{b c} \\
34,7^{d} \\
41,7^{c}\end{array}$ & $\begin{array}{l}45,8^{c} \\
86,4^{a} \\
66,1^{a}\end{array}$ & $\begin{array}{l}57,7^{\mathrm{b}} \\
52,4^{\mathrm{bc}} \\
55,0^{\mathrm{b}}\end{array}$ & $\begin{array}{l}49,4^{a} \\
42,4^{b}\end{array}$ \\
\hline $\begin{array}{l}\text { Taux } \\
\text { de } \\
\text { nouaison }\end{array}$ & $\begin{array}{l}\text { D-27 } \\
\text { D-23 } \\
\text { Moyenne }\end{array}$ & $\begin{array}{l}29,9^{b} \\
53,3^{d} \\
41,6^{c}\end{array}$ & $\begin{array}{l}30,4^{b} \\
55,2^{d} \\
42,8^{c}\end{array}$ & $\begin{array}{l}31,1^{\mathrm{b}} \\
64,7^{\mathrm{e}} \\
47,9^{\mathrm{d}}\end{array}$ & $\begin{array}{l}32,6^{b} \\
42,0^{c} \\
37,3^{c}\end{array}$ & $\begin{array}{l}28,2^{b} \\
13,6^{a} \\
20,9^{a}\end{array}$ & $\begin{array}{l}19,4^{\mathrm{a}} \\
32,5^{\mathrm{b}} \\
26,0^{\mathrm{b}}\end{array}$ & $\begin{array}{l}28,6^{\mathrm{a}} \\
43,5^{\mathrm{b}}\end{array}$ \\
\hline
\end{tabular}

Taux d'avortement des fleurs : 100 - [nombre de gousses au stade I/ nombre de fleurs) x 100]; taux d'avortement des gousses au stade I : 100 - [nombre de gousses au stade II / nombre de gousses au stade I) $\times 100$ ]; taux de nouaison (= complément à 100 du taux d'avortement) : (nombre de gousses au stade $\|$ / nombre de fleurs) $\times 100$.

apparaît, d'après le tableau II, que c'est essentiellement au niveau du passage fleursgousses au stade I que la différence entre les 2 génotypes est la plus importante (43,6\% pour D-27 contre $31,5 \%$ pour D23), mais cette différence reste cependant hautement significative, bien que moins forte, pour les taux d'avortement des gousses entre le stade I et le stade II $(49,4 \%$ contre $42,4 \%$ ).

Ces différences du niveau des avortements se traduisent au niveau des taux de nouaison par un avantage très hautement significatif de la lignée peu autofertile, dont le taux de nouaison est 1,5 fois supérieur à celui de la lignée autofertile. Globalement chez cette dernière, $71 \%$ des fleurs formées avortent, alors que $56 \%$ seulement le font chez la lignée peu autofertile. On peut tout de suite noter que ce taux d'avortement plus élevé de la lignée autofertile, compte tenu de sa production de fleurs si- gnificativement plus élevée, permet aux génotypes de produire des quantités de gousses par plante comparables.

En ce qui concerne l'effet traitement, pour le taux d'avortement des fleurs, le traitement pollinisation extérieure conduit au taux d'avortement statistiquement le plus élevé $(54,2 \%$; tableau II) pour la lignée autofertile, alors que pour la lignée peu autofertile c'est le traitement autopollinisation qui entraîne le taux d'avortement le plus important $(57,6 \%)$. Pour cette dernière lignée, $B$ hortorum apparaît comme le meilleur pollinisateur, avec un taux d'avortement de $14,4 \%$, significativement inférieur à celui de tous les autres traitements. On retrouve les mêmes conclusions pour les avortements des gousses au stade I, avec des taux d'avortement très élevés pour le traitement pollinisation extérieure chez la lignée autofertile $(57,7 \%)$, ainsi que pour le traitement autopollinisa- 
tion chez la lignée peu autofertile $(86,4 \%)$ et un bon comportement de $B$ pascuorum et de $B$ hortorum (respectivement $23,7 \%$ et $24,6 \%$ d'avortements). Enfin, au niveau des taux de nouaison, le traitement pollinisation extérieure apparaît inférieur aux autres traitements, de façon hautement significative pour la lignée autofertile. Chez la lignée peu autofertile, l'autopollinisation apparaît très nettement inférieure aux autres types de pollinisation $(13,6 \%$ de nouaison) alors que $B$ hortorum confirme sa supériorité à polliniser cette lignée (64,7\% de nouaison, différence hautement significative avec les autres traitements impliquant des insectes).

L'étude des figures 3 et 4 apporte des informations complémentaires sur les modalités d'avortement des organes sur la tige. Chez la lignée peu autofertile, il y a pour $B$ pascuorum et $B$ hortorum une parfaite superposition des courbes de formation des gousses au stade 1 et des gousses au stade II (fig $4 a$ et $4 b$ ). Cette superposition s'observe également pour le traitement déclenchement manuel, avec cependant un écart significativement plus grand entre les 2 courbes (fig 4d). Pour $B$ lapidarius, entre les étages 7 et 15 , le taux d'avortement des gousses au stade II est significativement plus important que celui des gousses au stade I, ce qui se traduit par un plus grand écart entre les 2 courbes (fig 4c). Cette différence est également importante à partir de l'étage 4 pour le traitement pollinisation extérieure (fig 4e), ainsi que pour le traitement autopollinisation (fig 4f). Chez la lignée autofertile, les taux d'avortements semblent moins dépendants du type de traitement de pollinisation. Pour tous les traitements, la production de gousses au stade II marque une diminution sensible après le $10 \mathrm{e}$ étage, bien que la production de gousses au stade I demeure inchangée jusqu'au $15^{\mathrm{e}}$ étage, sauf pour le traitement déclen- chement manuel et surtout pour le traitement extérieur. D'autre part, dans tous les traitements, l'écart entre les 2 courbes est très significatif.

\section{Influence sur le rendement et ses composantes}

L'analyse statistique des effets génotype et traitement pour le rendement grain par plante, ainsi que pour ses composantes, est récapitulée dans le tableau III.

Les effets génotype sont significatifs pour le nombre moyen de grains par plante $(101,6$ pour D-27 et 84,8 pour la lignée peu autofertile) ainsi que pour le rendement grain par plante (respectivement 41,2 et $31,8 \mathrm{~g}$ par plante). En autopollinisation, le nombre de grains par plante chez la lignée autofertile est peu différent de celui des autres traitements. II n'existe pas, globalement, de différences significatives entre les différentes espèces de bourdons.

Inversement, chez la lignée peu autofertile, en autopollinisation, le nombre de grains par plante est de 4 à 5 fois inférieur à celui obtenu dans les cages avec bourdons. Par ailleurs, les différentes espèces de bourdons produisent statistiquement plus de grains par plante que les autres traitements chez cette lignée.

En ce qui concerne le poids de 1000 grains, les différences ne sont pas significatives entre génotypes. II n'existe pas, d'autre part, de différences significatives entre traitements pour la lignée autofertile D-27. Pour la lignée peu autofertile, en autopollinisation, le poids de 1000 grains est très significativement supérieur à celui obtenu dans les autres traitements de pollinisation. Cette augmentation du poids de 1000 grains est corrélative du faible nombre de grains produits par la plante et traduit un phénomène de compensation entre 
Tableau III. Nombre moyen de grains par plante, poids moyen de 1000 grains et rendement moyen par plante chez les lignées $D-27$ et D-23 en fonction des traitements. Le rendement est exprimé en g/ plante.

\begin{tabular}{|c|c|c|c|c|c|c|c|c|}
\hline & & $\begin{array}{c}\text { B } \\
\text { lapidarius }\end{array}$ & $\begin{array}{c}\mathrm{B} \\
\text { pascuorum }\end{array}$ & $\underset{\text { hortorum }}{\text { B }}$ & $\begin{array}{l}\text { Déclenchement } \\
\text { n manuel }\end{array}$ & $\begin{array}{l}\text { nt Auto- } \\
\text { pollinisation }\end{array}$ & Extérieur & Moyenne \\
\hline $\begin{array}{l}\text { Nombre } \\
\text { moyen } \\
\text { de grains } \\
\text { par plante }\end{array}$ & $\begin{array}{l}\text { D-27 } \\
\text { D-23 } \\
\text { Moyenne }\end{array}$ & $\begin{array}{l}117,1^{a b} \\
108,8^{a b c} \\
112,9^{a b}\end{array}$ & $\begin{array}{l}107,7^{a b c} \\
112,3^{a b c} \\
110,0^{a b}\end{array}$ & $\begin{array}{c}114,6^{a b} \\
129,2^{a} \\
121,9^{a}\end{array}$ & $\begin{array}{c}110,1^{\text {abc }} \\
78,7^{\text {cde }} \\
94,4^{b}\end{array}$ & $\begin{array}{l}89,8^{\text {bcd }} \\
23,3^{1} \\
56,5^{c}\end{array}$ & $\begin{array}{l}70,5^{\text {de }} \\
57,1^{\text {e }} \\
63,8^{c}\end{array}$ & $\begin{array}{r}101,6^{\mathrm{a}} \\
84,8^{\mathrm{b}}\end{array}$ \\
\hline $\begin{array}{l}\text { Poids } \\
\text { moyen de } \\
1000 \text { grains } \\
\text { (g) }\end{array}$ & $\begin{array}{l}\text { D-27 } \\
\text { D-2 } \\
\text { Moyenne }\end{array}$ & $\begin{array}{l}388,5^{\mathrm{bc}} \\
342,6^{\mathrm{c}} \\
365,6^{\mathrm{c}}\end{array}$ & $\begin{array}{l}411,9 b c \\
371,7 b c \\
391,8 b c\end{array}$ & $\begin{array}{l}400,2^{\mathrm{bc}} \\
355,0^{\mathrm{c}} \\
377,6^{\mathrm{bc}}\end{array}$ & $\begin{array}{l}408,1^{b c} \\
440,3^{b} \\
424,2^{b}\end{array}$ & $\begin{array}{l}410,1^{\mathrm{bc}} \\
524,5^{\mathrm{a}} \\
467,3^{\mathrm{a}}\end{array}$ & $\begin{array}{l}424,1^{b c} \\
349,6^{c} \\
386,9^{b c}\end{array}$ & $\begin{array}{r}407,1 \\
397,3 \\
\text { (NS) }\end{array}$ \\
\hline $\begin{array}{l}\text { Rendement } \\
\text { en grains } \\
\text { par plante }\end{array}$ & $\begin{array}{l}\text { D-27 } \\
\text { D-23 } \\
\text { Moyenne }\end{array}$ & $\begin{array}{l}45,0^{a} \\
37,0^{a} \\
41,0^{a}\end{array}$ & $\begin{array}{l}44,3^{a} \\
41,7^{a} \\
43,0^{a}\end{array}$ & $\begin{array}{l}45,9^{a} \\
46,0^{a} \\
45,9^{a}\end{array}$ & $\begin{array}{l}45,0^{\mathrm{a}} \\
34,2^{\mathrm{a}} \\
39,6^{\mathrm{a}}\end{array}$ & $\begin{array}{l}37,3^{\mathrm{a}} \\
12,0^{\mathrm{b}} \\
24,6^{\mathrm{b}}\end{array}$ & $\begin{array}{l}30,0^{\mathrm{a}} \\
19,7^{\mathrm{b}} \\
24,8^{\mathrm{b}}\end{array}$ & $\begin{array}{l}41,2^{\mathrm{a}} \\
31,8^{\mathrm{b}}\end{array}$ \\
\hline
\end{tabular}

facteurs du rendement bien connu chez $V$ faba.

Enfin, pour le rendement en grains par plante, il n'y a, chez la lignée autofertile, aucune différence significative entre traitements. Inversement, chez la lignée peu autofertile, les traitements autopollinisation et pollinisation extérieure sont très significativement inférieurs aux autres traitements.

\section{DISCUSSION ET CONCLUSION}

Les 2 lignées, autofertile et peu autofertile, présentent une réaction différente vis-à-vis de la pollinisation. La lignée autofertile (D27) a une réaction homogène par rapport aux différents traitements, si l'on excepte le traitement en pollinisation libre, qui est inférieur aux autres pour le nombre moyen de grains et le rendement moyen par plante. Cette différence traduit un "effet cage" important qui pourrait être dû à l'effet de microclimat favorable à la plante, créé par la cage. En revanche, la lignée non autofertile (D-23) présente un effet traitement très hautement significatif. Si l'on excepte le traitement pollinisation extérieure pour les mêmes raisons que précédemment, on peut distinguer 3 effets :

- l'autopollinisation conduit à une chute considérable du nombre de grains et du rendement grainier par plante (diminution de plus de $75 \%$ du nombre de grains par rapport aux résultats obtenus en présence d'insectes) et à une compensation importante sur le poids moyen d'un grain;

- le déclenchement manuel produit significativement moins de grains que les traitements avec insectes, mais par compensation le poids moyen de 1000 grains est supérieur; il semblerait donc, dans ce cas, que les insectes agissent sur les fleurs de façon différente de celle provoquée par le simple déclenchement. Cette action positive pourrait traduire un rôle mécanique joué 
par l'insecte au niveau du stigmate permettant une pollinisation plus efficace; elle pourrait également traduire une forme partielle d'incompatibilité chez cette lignée, l'action positive de l'insecte par rapport au déclenchement manuel correspondant à l'apport d'allopollen de plantes voisines permettant de lever cette incompatibilité partielle;

- enfin, l'étude des profils de production de graines montre que les 3 espèces de bourdons étudiées n'ont pas une activité identique sur les différents niveaux des plantes; cependant, les différences s'observant au niveau des étages sur le développement des organes reproducteurs n'ont pas de conséquence sur le rendement final et ses composantes.

Par rapport à une expérimentation précédente (Mesquida et al, 1990), menée sur les mêmes génotypes, il apparaît quelques différences qui ne sont pas de nature à modifier nos conclusions. Les comparaisons sont possibles pour les traitements $B$ lapidarius, autopollinisation, déclenchement manuel et pollinisation extérieure communs dans les deux expérimentations (tableau IV).

Chez la lignée autofertile, on note dans le cas du traitement autopollinisation, pour le nombre de grains par plante et pour le rendement grain par plante, une stabilité remarquable pour les 2 expérimentations (respectivement 90,9 et 89,8 grains par plante en 1990 et en $1991 ; 39,9$ et $37,3 \mathrm{~g}$ de grains par plante). Le traitement déclenchement manuel donne pour cette lignée des résultats également homogènes pour ces 2 caractères $(94,4$ et 110,1 pour le nombre de grains par plante; 41,1 et $45,0 \mathrm{~g}$ de grains par plante). Le traitement pollinisation extérieure est également très stable ( 79,5 et 70,5 pour le nombre de grains par plante; 31,6 et $30,0 \mathrm{~g}$ de grains par plante respectivement pour les 2 années). Le traitement $B$ lapidarius donne en revanche des résultats supérieurs en 1991 par rapport à 1990, aussi bien pour le nombre de grains

Tableau IV. Comparaisons des résultats relatifs au rendement grain et à ses composantes dans l'expérimentation 1990 (résultats antérieurs) et celle de 1991 rapportés dans cette note. Le rendement est exprimé en grammes par plante. Le poids de 1000 grains est exprimée en grammes. D-27 : lignée autofertile; D-23 : lignée peu autofertile.

\begin{tabular}{|c|c|c|c|c|c|c|c|c|c|}
\hline & & \multicolumn{4}{|c|}{$D-27$} & \multicolumn{4}{|c|}{$D-23$} \\
\hline & & $\begin{array}{c}\text { B } \\
\text { lapidarius }\end{array}$ & $\begin{array}{c}\text { Auto- } \\
\text { pollinisation }\end{array}$ & Extérieur & $\begin{array}{l}\text { Déclen- } \\
\text { chement }\end{array}$ & $\begin{array}{c}\text { B } \\
\text { lapidarius }\end{array}$ & $\begin{array}{c}\text { Auto- } \\
\text { pollinisation }\end{array}$ & Extérieur & $\begin{array}{l}\text { Déclen- } \\
\text { chemen }\end{array}$ \\
\hline \multirow{2}{*}{$\begin{array}{l}\text { Nombre } \\
\text { de grains } \\
\text { par plante }\end{array}$} & 1990 & 80,0 & 90,9 & 79,5 & 94,4 & 80,2 & 8,6 & 78,2 & 71,9 \\
\hline & 1991 & 117,1 & 89,8 & 70,5 & 110,1 & 108,8 & 23,3 & 57,1 & 78,7 \\
\hline \multirow{2}{*}{$\begin{array}{l}\text { Poids } \\
\text { de } 1000 \\
\text { grains }\end{array}$} & 1990 & 416 & 441 & 401 & 436 & 418 & 658 & 387 & 457 \\
\hline & 1991 & 389 & 410 & 424 & 408 & 343 & 525 & 350 & 450 \\
\hline \multirow{2}{*}{$\begin{array}{l}\text { Poids } \\
\text { de grains } \\
\text { par plante }\end{array}$} & 1990 & 33,3 & 39,9 & 31,6 & 41,1 & 33,5 & 5,7 & 30,2 & 32,7 \\
\hline & 1991 & 45,0 & 37,3 & 30,0 & 45,0 & 37,0 & 12,0 & 19,7 & 34,2 \\
\hline
\end{tabular}


par plante $(80,0$ en 1990 contre 117,1 en 1991) que pour le rendement grain par plante $(33,3$ et $45,0 \mathrm{~g}$ respectivement).

Pour la lignée peu autofertile, seul le déclenchement manuel donne des résultats identiques pour le nombre de grains par plante et le poids de grain par plante $(71,9$ et 78,$7 ; 32,7$ et 34,2 respectivement pour 1990 et 1991).

Ces résultats confirment que la production grainière chez la féverole est fonction de la biologie florale du cultivar considéré. Les insectes dans le cas de la lignée autofertile D-27 n'augmentent pas le rendement, encore que l'effet "année" observé pour $B$ lapidarius entre 1990 et 1991 mérite d'être précisé. II n'en va pas de même pour la lignée non autofertile D-23, chez laquelle la pollinisation par les insectes est obligatoire pour assurer une production optimale de grains. Ces résultats montrent également que la pollinisation par les insectes ne semble pas être un facteur limitant de la production de grains chez la féverole, du moins pour la lignée autofertile. Ces résultats soulèvent toutefois des questions d'ordre fondamental.

Il est nécessaire de comprendre ce qui fait, au plan physiologique la différence entre une lignée autofertile et une lignée non autofertile ou peu autofertile. II n'y a pas en effet, au plan morphologique, de différence entre fleur autofertile et fleur non autofertile (Le Guen, non publié). II est possible que la différence se situe au plan génétique et traduise certaines formes d'incompatibilité déjà montrée au sein du groupe d'espèces Vicia faba (Le Guen, 1983). L'autofertilité pourrait alors correspondre à une levée partielle ou complète de cette incompatibilité chez certains cultivars. Le phénomène doit, en fait, être plus complexe, car nous avons montré par ailleurs (Mesquida et al, 1990; Carre et al, 1991) que la lignée autofertile D-27 est moins réceptive à l'allopollen que la lignée non autofertile D-23, ainsi qu'en témoignent les taux d'intercroisement plus faibles chez la première lignée que chez la seconde.

Par ailleurs, chez la lignée non autofertile, les plantes peuvent produire, en conditions d'autopollinisation, une certaine quantité de graines. Cette production, très faible sur les premiers nœuds floraux, s'accroît ensuite, sans jamais atteindre toutefois le niveau obtenu en conditions de pollinisation par les insectes ou par le déclenchement. Ce phénomène implique une modification de la biologie florale de la lignée. II serait important de connaître la nature de cette modification pour améliorer notre compréhension des mécanismes de mise à fruit chez la féverole. Plusieurs hypothèses peuvent être avancées, telle qu'une hypothèse génétique faisant intervenir la levée partielle d'une barrière d'incompatibilité, ou une hypothèse métabolique telle que l'inversion d'un trophisme entre puits et source métaboliques. Audelà de la cause de cette inversion, qu'elle soit génétique ou trophique, la nature du stimulus qui la déclenche est fondamentale à connaître. On peut proposer des voies de recherches en ce sens impliquant par exemple les variations de température existant au cours de la très longue phase de floraison de la féverole, variations dont on sait qu'elles ont une influence sur les phénomènes d'incompatibilité, mais également sur les translocations métaboliques dans les plantes.

La production de fleurs correspond à une dépense énergétique importante chez une plante, et un avortement de fleurs peut être considéré comme une perte potentielle de productivité. L'autofertilité s'accompagne de taux d'avortement de fleurs et de gousses significativement supérieurs à ceux exprimés par la lignée non autofertile. Cela se traduit par un taux de nouaison pratiquement 2 fois inférieur pour le type 
autofertile. Par conséquent, dans le cadre du matériel analysé dans cette étude, l'autofertilité entraîne globalement une limitation du potentiel de rendement mis en place par la lignée au "stade fleurs" (127,5 fleurs formées chez D-27 contre 84,1 chez D-23 pour un rendement équivalent chez les 2 lignées). Nous n'avons pas actuellement d'explication à ce phénomène. On peut cependant formuler I'hypothèse que la production d'un grand nombre de fleurs correspond à la mise en place d'un puits métabolique reproducteur intense permettant de détourner les translocats vers les organes reproducteurs en limitant les effets de la dominance apicale, très importante, normalement exercée par le bourgeon terminal végétatif dans cette espèce à croissance indéterminée. L'autofertilité pourrait dans ce cas être le résultat de ce rééquilibrage dans les relations de dominance apex/organes reproducteurs latéraux. II serait intéressant de tester cette hypothèse à partir de la comparaison de lignées parfaitement isogéniques autofertiles et non autofertiles, à condition que l'on puisse les obtenir.

Il faut enfin noter que l'efficacité pollinisatrice de $B$ hortorum a été sensiblement meilleure que celle de $B$ lapidarius et de $B$ pascuorum aussi bien au niveau des taux de nouaison qu'à celui du rendement en grains par plante. On peut penser que ce résultat est lié au fait que $B$ hortorum possède un proboscis beaucoup plus long que celui des autres espèces de bourdons utilisées (Pouvreau, 1984), ainsi qu'une activité pollinisatrice et une vitesse de butinage nettement supérieures (Tasei, 1976). D'après les valeurs rapportées par Pouvreau, les fondatrices de $B$ hortorum ont un proboscis de grande taille $(18,0$ à 21,2 $\mathrm{mm})$, alors que celui de $B$ lapidarius et de $B$ pascuorum est de plus petite taille (12,0 à $14,8 \mathrm{~mm}$ ). La longueur du proboscis des bourdons est généralement mise en rela- tion avec la nature et la profondeur de la corolle des fleurs visitées (Free, 1962; Pouvreau, 1985), mais cela ne semble pas se vérifier dans tous les cas. De même, selon Pouvreau (1984, 1985), cela ne suffit pas à expliquer les préférences dans la diversité des fleurs visitées. II existerait également une certaine relation entre la taille du proboscis de ces espèces et le coefficient d'efficacité pollinisatrice de la féverole (Tasei, 1976). Chez les bourdons, ce coefficient en conditions de plein champ est très supérieur à celui de l'abeille domestique. Cependant, l'efficacité réduite de cette dernière sur la féverole est en grande partie due aux visites négatives consécutives à l'utilisation de trous percés à la base de la corolle par $B$ terrestris et à l'exploitation des nectaires extrafloraux portés par les stipules foliaires (Brandenburg, 1961; Free, 1962; Poulsen, 1973; Tasei, 1976). D'autres espèces du groupe Bombus à langue très courte adoptent également ce type de butinage négatif.

En conclusion, on peut souligner que la lignée autofertile produit nettement plus de fleurs que la lignée non autofertile et présente des taux d'avortement significativement plus élevés. Elle est indifférente aux types de pollinisation et à l'espèce de l'insecte pollinisateur. Cela se traduit par des taux d'avortement et des rendements globablement identiques dans toutes les situations. Par ailleurs, malgré un taux d'avortement plus élevé, cette lignée autofertile a pour tous les traitements une productivité supérieure à celle de la lignée non autofertile. Enfin, le fait que la lignée autofertile soit indépendante des conditions de pollinisation et de la présence d'insectes pollinisateurs pour assurer son rendement montre que l'autofertilité chez la féverole peut être un moyen naturel de régulariser et de stabiliser le niveau des rendements, mais elle ne permet pas de les augmenter. 
La lignée non autofertile, en revanche, est plus sensible à l'espèce de bourdon et a obligatoirement besoin d'insectes pollinisateurs pour accroittre la nouaison et le rendement.

\section{Summary - Efficiency of pollinating} treatments on 2 lines of spring faba bean (Vicia faba $L$ var equina Steudel) with different levels of self-fertility and using 3 species of Bombus (Hymenoptera: Apidae). The objective was to compare the pollinating efficiency of Bombus lapidarius, $B$ pascuorum and $B$ hortorum with 3 types of treatments (hand tripping, self pollination and open pollination). This study was conducted under insect-proof cages $3 \times 3 \times 3 \mathrm{~m}$ on 2 faba bean lines, one with high self-fertility (D-27) and the other with low self-fertility (D-23). Another objective of the study was to further evaluate if self-fertility might increase the reliability of yield in faba bean. The cages with bumble bees contained 2 queens each, and the experimental design was a randomized complete block with 3 replications, 6 pollination treatments and 2 lines per treatment. Data were recorded on reproductive organs (flowers and pods) by node, on abortion rates, pod setting rates and seed yield components. Treatments did not affect flower production, the selffertile line $D-27$ yielding $\approx 50 \%$ more flowers than the line D-23 with low self-fertility. But the abortion rates were significantly higher in the self-fertile line D-27 than in D-23.

The self-fertile line was not affected by the various conditions of pollination. On the contrary, the line with low self-fertility was very susceptible to treatments and to the different species of bumble bees. Under self pollination, the low self-fertile line produced significantly less pods than with insects. On the contrary self-fertility enabled line D-27 to produce as many seeds under self-pollination as with bumble bees. Despite its higher abortion rate, the selffertile line $\mathrm{D}-27$ had a greater productivity than that of D-23 for practically all the treatments. We concluded that the non self-fertile line has a definite need of pollinating insects to significantly increase its pod setting and seed yield. The fact that the yield from the self-fertile line was completely independent of insect activity indicates that self fertility was an effective means of making faba bean yield more consistent and reliable, but this mechanism did not increase yield.

Vicia faba / Bombus / insect pollination / self-pollination / seed yield

\section{Zusammenfassung - Die Bestäubung} der frühjahrsblühenden Pferdebohne (Vicia faba L var equina Staudel). Die Bestäubunsgsleistung von 3 Hummelarten (Bombus Latr, Hymenoptera : Apidae) bei zwei Linien der Pferdebohne mit unterschiedlichem Grad der Selbstfertilität. Das Ziel der Untersuchung war ein Vergleich der Bestäubungsleistung von Bombus lapidarius, $B$ pascuorum und $B$ hortorum mit drei Versuchsanordnungen (Auslösung der Blüte ("tripping") von Hand, Selbstbestäubung und offene Bestäubung). Die Versuche wurden in insektendichten Käfigen $3 \times 3 \times$ $3 \mathrm{~m}$ mit zwei Linien der Pferdebohne durchgeführt, eine mit hoher Selbstfertilität (D-27), die andere mit niedriger Selbstfertilität (D-23). Ein weiteres Versuchsziel war eine weitere Klärung der Frage, ob Selbstfertilität die Verläßlichkeit der Erträge bei der Pferdebohne steigern könnte.

Die Käfige mit Hummeln enthielten je zwei Königinnen. Die Versuchsanordnung bestand aus einem randomisierten vollständigen Block mit drei Wiederholungen, sechs Bestäubungsarten und zwei Linien 
pro Einzelversuch. Es wurden Daten über die Fortpflanzungsorgane (Blüten und Schoten) je Wirtel registriert, sowie über die Rate unentwickelter Samen (Abortion), die Rate des Schotenansatzes und über den Samenertrag.

Die verschiedenen Behandlungen beeinflußten den Blütenansatz nicht; die selbstfertile Linie D-27 brachte etwa 50\% mehr Blüten als die Linie D-23 mit niedriger Selbstfertilität. Aber die Abortionsraten waren bei der selbstfertilen Linie D-27 signifikant höher als bei D-23. Die selbstfertile Linie wurde durch die verschiedenen Bestäubungsbedingungen nicht beeinflußt. Dagegen erwies sich die Linie mit niedriger Selbstfertilität als sehr empfindlich gegenüber den Versuchsanordnungen und den verschiedenen Hummelarten. Bei Selbstbestäubung erzeugte die wenig selbstfertile Linie signifikant weniger Schoten als bei Anwesenheit von Insekten. Dagegen ermöglichte es die Selbstfertilität der Linie D-27 unter den Bedingungen der Selbstbestäubung ebensoviele Samen zu erzeugen wie mit Hummeln. Trotz ihrer höheren Abortionsrate zeigte die selbstfertile Linie D-27 praktisch unter allen Versuchsbedingungen eine höhere Produktivität als die Linie D-23.

Wir zogen daraus den Schluß, daß die nicht-selbstfertile Linie zur signifikanten Erhöhung des Schotenansatzes und des Samenertrages definitiv der Insektenbestäubung bedarf. Die Tatsache, daß die selbstfertile Linie zur Sicherung der Ernte vollständig von der Aktivität von Insekten unabhängig war, deutet darauf hin, daß Selbstfertilität ein wirksames Mittel darstellt, um die Ernten der Pferdebohme beständiger und verläßlicher zu machen, ohne dadurch jedoch die Ernten zu erhöhen.

\section{Bombus sp / Pferdebohne / Vicia faba / Bestäubung / Samenertrag / Selbstbe- stäubung}

\section{RÉFÉRENCES}

Berthélem P (1966) Essai d'utilisation d'insectes pollinisateurs (Bombus Latr) dans l'amélioration de la féverole (Vicia faba L). Ann Amelior Plant 16, 101-115

Brandenburg W (1961) Broad beans: causes of poor yield sought. $N Z$ J Agric 102, 277-280

Carré S, Taséi JN, Mesquida J, Le Guen J (1991) Estimate of outcrossing rate between 2 lines of field beans (Vicia faba $L$ ) in various conditions with isozymic markers. Acta Hortic 288, 354-358

Cooper BA (1964) Pollination of field bean. $\mathrm{Br}$ Bee J 92/3, 102-103

Free JB (1962) The behaviour of honey bees visiting field beans (Vicia faba). J Anim Ecol 31, 497-502

Free JB (1966) Pollination requirement of broad beans and field beans (Vicia faba). J Agric Sci 66, 395-397

Gouet H, Gouet JP, Philippeau G, Tranchefort J, Verneau M (1985) Manuel d'utilisation STAT ITCF. Service des études statistiques et informatiques de IITCF, Boigneville, II 8.1-8.13

Le Guen J (1983) Incompatibilité unilatérale chez Vicia faba L. Analyses globales de croisements intraspécifiques entre 4 sousespèces. Agronomie 3/5, 443-449

Lerin J (1982) Effets de la pollinisation entomophile sur le colza dans une expérience en cage. Agronomie 2, 249-256

Link W (1990) Autofertility and rate of crossfertilization: crucial characters for breeding synthetic varieties in faba beans (Vicia faba L). Theor App/ Genet 79, 713-717

Martyanova Al (1967) Effect of the pollination method on the quality and productivity of field bean sees (in Russian). Bull Moscow Soc Nat Biol Sect 72, 83-92

Mesquida J, Renard M, Pierre JS (1988) Rapeseed (Brassica napus $L$ ) productivity: the effect of honey bees (Apis mellifera L) and different pollination conditions in cage and field tests. Apidologie 19, 51-72

Mesquida J, Le Guen J, Taséi JN, Carré S, Morin G (1990) Modalités de la pollination chez 2 lignées de féverole de printemps (Vicia faba $L$ var equina Steudel). Effets sur 
les coulures, la productivité et les taux de croisements. Apidologie 21, 511-525

Poulsen MH (1971) Honnigbiernes (Apis L) og humlebiernes (Bombus Latr) arbejdsmade og betydning for frosaetningen i hestebonne (Vicia faba L). Royal Veterinary and Agric University Copenhagen Thesis

Poulsen MH (1973) The frequency and foraging behaviour of honeybees and bumble bees on field beans in Denmark. J Apic Res 12, 75-80

Poulsen MH (1975) Pollination, seed setting, cross fertilization and inbreeding in Vicia faba L. $Z$ Pflanzenzüchtg 74, 97-118

Pouvreau $A$ (1965) Sur une méthode d'élevage des bourdons (Bombus Latr) à partir de reines capturées dans la nature. Ann Abeille 8, 147-159

Pouvreau A (1984) Biologie et écologie des bourdons. In : Pollinisation et productions végetales (Pesson P, Louveaux L, eds) INRA, Paris, 595-630

Pouvreau A (1985) Adaptations morphologiques à la récolte du nectar chez les bourdons. Incidences sur l'activité de butinage (Hymenoptera, Apidae, Bombinae). Actes Colloq Insectes Soc 2, 65-77
Pouvreau A, Marilleau R (1977) L'élevage des bourdons. Leur utilisation pour la pollinisation des plantes. Cah Liaisons OPIE 25, 22-27

Pritsch G (1971) Recherches sur le rôle que joue l'abeille dans la pollinisation de la fève (Vicia faba). C R 23e Congr Int Apic Moscou, Bucarest, Apimondia, 529-530

Riedel IBM, Wort DA (1960) The pollination requirement of the field bean (Vicia faba). Ann Appl Biol 48, 121-124

Scriven WA, Cooper BA, Allen H (1961) Pollination of field beans. Outlook Agric 3, 69-75

Taséi JN (1976) Les insectes pollinisateurs de la féverole d'hiver (Vicia faba equina $L$ ) et la pollinisation des plantes mâles stériles en production de semences hybrides. Apidologie 7, 1-38

Wafa AK, Ibrahim SH (1960) The effect of the honey bee as a pollinating agent on the yield of broad bean. Bull Fac Agric Ain Shams Univ 205, 3-35

Watts FH, Marshall PR (1961) Pollination of field beans. Yield response due to bees. Report on field experiments and observation studies, East Midland Region, 76-79 